\title{
DIFFEOMORPHISMS OF MANIFOLDS WITH FINITE FUNDAMENTAL GROUP
}

\author{
GEORGIA TRIANTAFILLOU
}

\begin{abstract}
We show that the group $\mathscr{D}(M)$ of pseudoisotopy classes of diffeomorphisms of a manifold of dimension $\geq 5$ and of finite fundamental group is commensurable to an arithmetic group. As a result $\pi_{0}($ Diff $M)$ is a group of finite type.
\end{abstract}

Let $M$ be an $n$-dimensional closed smooth manifold, where $n \geq 5$, and let DiffM be the group of diffeomorphisms of $M$. The space DiffM (it is a topological space with the $C^{\infty}$-topology) is in general very complicated and has been studied by many authors. Its homotopy type is known only in some special cases. We recall the well-known results $\operatorname{Diff}\left(S^{2}\right) \simeq O(3)$ by Smale $[\mathrm{Sm}]$ and $\operatorname{Diff}\left(S^{3}\right) \simeq O(4)$ by Hatcher $[\mathrm{H}]$.

The component group $\pi_{0}($ Diff $M)$ has also been initially computed in special cases, for instance for spheres, where the group $\pi_{0}\left(D i f f_{+} S^{n}\right)$ of orientationpreserving diffeomorphisms is isomorphic to the group of homotopy spheres of dimension $n+1$ for $n>4[\mathrm{KM}$ ], and for products of spheres [B, Tu]. The first general result about the nature of $\pi_{0}($ Diff $M)$ for simply connected manifolds $M$ is due to Sullivan [S], who showed that if $M$ is a smooth closed orientable simply connected manifold of dimension $\geq 5$, then $\pi_{0}($ Diff $M)$ is commensurable to an arithmetic group.

Arithmetic groups and their properties have been studied in [BH] by Borel and Harish-Chandra. In particular they showed that every arithmetic group is finitely presented. It follows that $\pi_{0}(D i f f M)$ is finitely presented if $M$ is simply connected. In fact $\pi_{0}$ (Diff $\left.M\right)$ in this case is a group of finite type by a result of Borel and Serre that arithmetic groups are of finite type [BS]. By definition a group $\pi$ is of finite type if its classifying space $B \pi$ is homotopy equivalent to a CW-complex with finitely many cells in each dimension. Being of finite type for a group implies and in fact is much stronger than finite presentation. Two groups are said to be commensurable if there is a finite sequence of homomorphisms between them which have finite kernels and images of finite index.

On the other hand $\pi_{0}($ DiffM $)$ can be very large if $M$ is not simply connected. For instance, $\pi_{0}\left(D_{i f f} T^{n}\right)$ contains a subgroup isomorphic to a direct sum of infinitely many copies of $\mathbb{Z}_{2}$ for $n \geq 5$, and $\pi_{0}\left(\operatorname{Diff}\left(T^{n} \times S^{2}\right)\right)$ contains a free abelian subgroup of infinite rank for $n \geq 3$, where $T^{n}$ is the torus [HS]. In these cases $\pi_{1}(M)$ is infinite.

In this paper we study $\pi_{0}(\operatorname{Diff} M)$ and $\mathscr{D}(M)$ in the case where $\pi_{1}(M)$ is

Received by the editors September 30, 1993.

1991 Mathematics Subject Classification. Primary 57R50, 57R52, 57S05; Secondary 57R67, $55 \mathrm{P} 62$.

Key words and phrases. Diffeomorphism, isotopy, arithmetic group, homotopy equivalence. 
finite, where $\mathscr{D}(M)$ is the group of pseudoisotopy classes of diffeomorphisms of $M$.

Pseudoisotopy is defined by dropping the level preserving requirement of isotopy, namely, two diffeomorphisms $f$ and $g$ of $M$ are pseudoisotopic if there is a diffeomorphism $F: M \times I \rightarrow M \times I$ which restricts to $f$ and $g$ at the two ends of $M \times I$ respectively. While in the simply connected case $\pi_{0}($ Diff $M)=\mathscr{D}(M)$ if $n \geq 5$ by Cerf's result [C], in the nonsimply connected case there is in general an Abelian kernel of the forgetful map

$$
0 \rightarrow A \rightarrow \pi_{0}(\text { Diff } M) \rightarrow \mathscr{D}(M) \rightarrow 0,
$$

where $A$ has been computed by Hatcher and Wagoner [HW] and Igusa [I]. It is also well known to the experts that $A$ is finitely generated if $\pi_{1}(M)$ is finite. We show:

Theorem 1. Let $M$ be a closed, smooth, orientable manifold of dimension $\geq 5$ with finite fundamental group. Then the group $\mathscr{D}(M)$ of pseudoisotopy classes of diffeomorphisms of $M$ is commensurable to an arithmetic group.

As a consequence of this and the exact sequence above we get:

Theorem 2. If $M$ is a smooth, closed, orientable manifold of dimension $\geq 5$ with finite fundamental group, then $\pi_{0}($ Diff $M)$ is of finite type.

\section{OUTLINE OF PROOFS}

The proof breaks up naturally into two parts: a homotopy theoretical part and a geometric part. Let $\operatorname{aut}(M)$ be the group of homotopy classes of selfhomotopy equivalences of $M$, and let $a u t_{t}(M) \subseteq \operatorname{aut}(M)$ be the subgroup, the elements of which are represented by maps $f$ which preserve the tangent bundle, i.e., $f^{*}(T(M))=T(M)$. First we show that the group aut $(M)$ is commensurable to an arithmetic group. For this purpose we employ minimal model techniques in the equivariant context and certain facts from the theory of algebraic and arithmetic groups. In the presence of a finite fundamental group we work with the minimal model of the universal cover of $M$, where the model is now equipped with an action of $\pi_{1}$. By using this equivariant minimal model, we show the following.

Theorem 3. The group aut $(M)$ of homotopy classes of self-homotopy equivalences of a finite $C W$-complex $M$ with finite fundamental group is commensurable to an arithmetic group.

This generalizes a result of [W] for the simply connected case and independently of [S] for the nilpotent case. It has been shown by [DDK] that aut $(X)$ is of finite type for virtually nilpotent spaces including spaces with finite fundamental group. The full strength of arithmeticity of aut $(X)$ above is needed to prove the following result. We note here that the properties of being of finite type, finitely presented, finitely generated, or arithmetic are not in general inherited by subgroups.

Theorem 4. The group aut $t_{t}(M)$ of homotopy classes of tangential self-homotopy equivalences of a manifold $M$ with finite fundamental group is commensurable to an arithmetic group. 
In the geometric part of the proof we apply surgery techniques and the AtiyahSinger $G$-signature theorem to relate the group of tangential homotopy equivalences to the group diff $M$ of homotopy classes of diffeomorphisms, where the latter is the image of $\pi_{0}$ (DiffM) into the group of homotopy classes of selfhomotopy equivalences. Out of this comparison follows in particular:

Theorem 5. The group of homotopy classes of tangential simple self-homotopy equivalences aut $t_{t}^{s}(M)$ is commensurable to an arithmetic group if $M$ has finite fundamental group and dimension $\geq 5$.

Further, we relate bundle homotopy classes of tangential homotopy equivalences to pseudoisotopy classes of diffeomorphisms. Let $\operatorname{Aut}\left(M, t_{0}\right)$ be the set (monoid) of self-homotopy equivalences of $M$ which preserve the tangent bundle except at a base point $x_{0} \in M$. More precisely, the elements of $\operatorname{Aut}\left(M, t_{0}\right)$ are pairs $(h, b)$, where $h$ is a base point preserving self-homotopy equivalence of $M$ and $b: T\left(M-x_{0}\right) \rightarrow T\left(M-x_{0}\right)$ is a bundle map covering $h$. Let $\operatorname{aut}\left(M, t_{0}\right)=\pi_{0}\left(\operatorname{Aut}\left(M, t_{0}\right)\right)$ be the group of bundle homotopy classes of such pairs.

The following is the main geometric result leading to the arithmeticity of $\mathscr{D}(M)$ as it relates the latter to homotopy and hence algebraic data. Under the same conditions as above we have:

Theorem 6. The group of pseudoisotopy classes of diffeomorphisms $\mathscr{D}(M)$ is commensurable to the group aut $\left(M, t_{0}\right)$ of bundle homotopy classes of selfhomotopy equivalences covered by bundle maps of the tangent bundle except at one point.

These groups appear as arithmetic subgroups of an algebraic matrix group over the rationals involving isomorphisms of the minimal model provided with certain additional structure.

Details of the proofs will appear elsewhere.

\section{ACKNOWLEDGMENTS}

I would like to thank Shmuel Weinberger for useful discussions and the University of Chicago for its hospitality while part of this research was done.

\section{REFERENCES}

[B] W. Browder, Diffeomorphisms of 1-connected manifolds, Trans. Amer. Math. Soc. 128 (1967), 155-163.

[BH] A. Borel and Harish-Chandra, Arithmetic subgroups of algebraic groups, Ann. of Math. (2) 75 (1962), 485-535.

[BS] A. Borel and J. P. Serre, Corners and arithmetic groups, Comment. Math. Helv. 48 (1973), 436-491.

[C] J. Cerf, La stratification naturelle des espaces de fonctions différentiables réelles et le theoréme de la pseudoisotopie, Publ. Math. Inst. Hautes Études Sci. 39 (1970).

[DDK] E. Dror, W. Dwyer, and D. Kan, Self homotopy equivalences of virtually nilpotent spaces, Comment. Math. Helv. 56 (1981), 599-614.

[H] A. Hatcher, A proof of a Smale conjecture, Ann. of Math. (2) 117 (1983), 553-607.

[HW] A. Hatcher and J. Wagoner, Pseudo-isotopies of compact manifolds, Asterisque 6 (1973).

[HS] W. C. Hsiang and R. Sharpe, Parametrized surgery and isotopy, Pacific J. Math. 67 (1976), 401-459. 
[I] K. Igusa, What happens to Hatcher and Wagoner's formula for $\pi_{0}(C(M))$ when the first Postnikov invariant of $M$ is nontrivial?, Lecture Notes in Math., vol. 1046, SpringerVerlag, New York, 1984, pp. 104-172.

[KM] M. Kervaire and J. Milnor, Groups of homotopy spheres. I, Ann. of Math. 2 (1963), 504-537.

[Sm] S. Smale, Diffeomorphisms of the 2-sphere, Proc. Amer. Math. Soc. 10 (1969), 621-626.

[S] D. Sullivan, Infinitesimal computations in topology, Publ. Math. Inst. Hautes Études Sci. 47 (1978), 269-331.

[Tu] E. Turner, Diffeomorphisms of a product of spheres, Invent. Math. 8 (1969), 69-82.

[W] C. Wilkerson, Minimal simplicial groups, Topology 15 (1976), 111-130.

Department of Mathematics, Temple University, Philadelphia, Pennsylvania 19122

E-mail address: georgia@euclid.math.temple.edu 